\title{
Andreev Transport through a Magnetic Molecule Weakly Coupled to Ferromagnetic Leads
}

\author{
F. PAWLICKI AND I. WEYMANN* \\ Faculty of Physics, Adam Mickiewicz University, Umultowska 85, 61-614 Poznań, Poland
}

\begin{abstract}
The Andreev transport through a single molecular magnet coupled to two external ferromagnetic leads and one superconducting electrode is studied theoretically by means of the real-time diagrammatic technique. The calculations are performed by including the sequential tunneling processes between the molecule and ferromagnetic leads, while the coupling to superconductor can be arbitrary. We analyze the dependence of the Andreev current and tunnel magnetoresistance on various intrinsic parameters of the molecule. The superconducting proximity effect results in the formation of molecular Andreev bound states. We show that the transport behavior depends greatly on the type of internal exchange interaction of the molecule, which can lead to corresponding sign changes of the tunnel magnetoresistance of the device.
\end{abstract}

DOI: 10.12693/APhysPolA.133.594

PACS/topics: 73.23.-b, 74.45.+c, 75.50.Xx

\section{Introduction}

Transport properties of magnetic molecules, such as single-molecular magnets (SMMs), attached to external leads have recently attracted considerable attention [1]. This is due to the fact that such nano-scale objects have great potential for applications in molecular electronics and spintronics. Besides, they offer a unique playground for testing various fundamental correlations and interactions between spins and charges. Theoretical investigations of transport properties of magnetic molecules have so far mainly focused on molecules attached to normal electrodes, both nonmagnetic and ferromagnetic [2 3]. In this paper we extend these studies and analyze the transport behavior of a molecular junction with ferromagnetic and superconducting electrodes. In such a hybrid system, in the sub-gap transport regime, the current flows due to Andreev reflection processes occurring through molecular Andreev bound states triggered by the superconducting proximity effect. We analyze the bias voltage dependence of the Andreev current on intrinsic parameters of the molecule. We also predict a nontrivial behavior of the tunnel magnetoresistance (TMR), depending on the bias voltage and the type of internal exchange interaction of the molecule.

\section{Theoretical description}

We consider a magnetic molecule coupled symmetrically to two ferromagnetic leads and one $s$-wave superconducting electrode, see Fig. 1. The magnetic moments of ferromagnetic electrodes are assumed to form either parallel $(P)$ or antiparallel $(A P)$ configuration. The voltage drop is applied between the superconductor (assumed to be grounded) and ferromagnetic leads kept at equal

\footnotetext{
*corresponding author; e-mail: weymann@amu.edu.pl
}

chemical potentials, $\mu_{L}=\mu_{R}=e V$. In our investigations we focus on the subgap transport regime, where current flows exclusively due to Andreev reflection processes. It is also assumed that transport occurs through the lowest unoccupied molecular orbital (LUMO) of the molecule, which is exchange coupled (with strength $J$ ) to the magnetic core spin $\boldsymbol{S}$ of SMM with uniaxial anisotropy $D[3]$. Under such assumptions, the system can be modeled by the following effective Hamiltonian

$$
H=H_{S M M}^{\mathrm{eff}}+H_{F M}+H_{T},
$$

where the first term describes the proximized singlemolecular magnet

$$
\begin{aligned}
& H_{S M M}^{\mathrm{eff}}=\varepsilon n+U n_{\uparrow} n_{\downarrow}-D S_{z}^{2}-J \boldsymbol{s} \cdot \boldsymbol{S} \\
& -\frac{\Gamma_{S}}{2}\left(d_{\uparrow}^{\dagger} d_{\downarrow}^{\dagger}+d_{\downarrow} d_{\uparrow}\right) .
\end{aligned}
$$

Here, $n_{\sigma}=d_{\sigma}^{\dagger} d_{\sigma}$ and $n=n_{\uparrow}+n_{\downarrow}$, with $d_{\sigma}^{\dagger}$ being the creation operator of an electron with spin $\sigma$ and energy $\varepsilon$ in the LUMO level, $U$ is the Coulomb repulsion energy, while $s$ is the spin operator of electrons in the LUMO level. The last term, proportional to $\Gamma_{S}$, accounts for the proximity-induced pairing potential in the SMM [4].

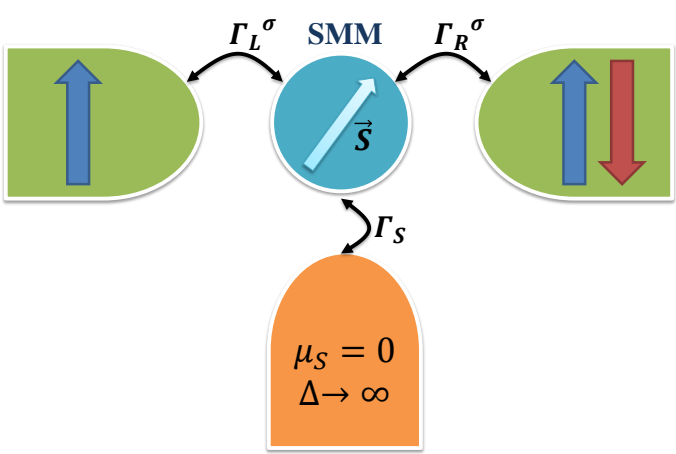

Fig. 1. Schematic of the considered hybrid molecular junction: a single molecular magnet (SMM) coupled to superconducting and ferromagnetic electrodes. 
The second term of the total Hamiltonian describes the ferromagnetic leads in the free quasi-particle approximation, $H_{F M}=\sum_{\beta \boldsymbol{k} \sigma} \varepsilon_{\beta \boldsymbol{k} \sigma} c_{\beta \boldsymbol{k} \sigma}^{\dagger} c_{\beta \boldsymbol{k} \sigma}$, where $c_{\beta \boldsymbol{k} \sigma}^{\dagger}$ is the creation operator of an electron with spin $\sigma$, momentum $\boldsymbol{k}$ and energy $\varepsilon_{\beta \boldsymbol{k} \sigma}$ in the lead $\beta$, with $\beta=L, R$ for the left/right electrode. The last term of Eq. (1) accounts for tunneling between the molecule and ferromagnetic leads and has the form, $H_{T}=\sum_{\beta \boldsymbol{k} \sigma} V_{\beta \boldsymbol{k} \sigma}\left(c_{\beta \boldsymbol{k} \sigma}^{\dagger} d_{\sigma}+\right.$ H.c. $)$, where $V_{\beta \boldsymbol{k} \sigma}$ are the relevant tunneling amplitudes. The effective coupling between SMM and ferromagnetic leads can be described by $\Gamma_{\beta}^{\sigma}=2 \pi\left|V_{\beta \sigma}\right|^{2} \rho_{\beta}^{\sigma}$, where $\rho_{\beta}^{\sigma}$ denotes the spin-resolved density of states of the respective lead and tunnel matrix elements were assumed to be momentum independent. In the following we assume that the system is symmetric, $\Gamma_{L}=\Gamma_{R} \equiv \Gamma / 2$, with $\Gamma_{\beta}=\Sigma_{\sigma} \Gamma_{\beta}^{\sigma}$, and introduce the spin polarization of the leads, $p_{L}=p_{R} \equiv p$.

The analysis of transport characteristics is performed by employing the real-time diagrammatic technique $[5,6]$. Assuming weak coupling between the molecule and ferromagnetic leads, we perform a systematic perturbative expansion with respect to the coupling strength $\Gamma$. In our considerations we include the lowestorder terms of expansion, which correspond to sequential tunneling. The steady-state occupation probabilities $P_{\chi}$ of the eigenstates $|\chi\rangle$ of the Hamiltonian (2) can be found from $\Sigma_{\chi^{\prime}} \Sigma_{\chi, \chi^{\prime}} P_{\chi^{\prime}}=0$, together with $\Sigma_{\chi} P_{\chi}=1$. Here, $\Sigma_{\chi, \chi^{\prime}}$ is the self-energy describing transitions between the corresponding states $\left|\chi^{\prime}\right\rangle$ and $|\chi\rangle$. The current flowing through the junction $\beta$ with ferromagnetic lead is given by the formula $I_{\beta}=-\frac{\mathrm{i} e}{2 \hbar} \sum_{\chi \chi^{\prime}} \Sigma_{\chi, \chi^{\prime}}^{I} P_{\chi}$, where $\Sigma_{\chi, \chi^{\prime}}^{I}$ is the self-energy that takes into account the number of electrons that tunneled through a given junction. The Andreev current flowing between superconductor and ferromagnetic leads can be then simply found using the Kirchhoff law, $I_{S}=I_{L}+I_{R}$.

\section{Results and discussion}

In the following we are interested in the behavior of the Andreev current in both the parallel $\left(I_{S}^{P}\right)$ and antiparallel $\left(I_{S}^{A P}\right)$ configuration of ferromagnetic leads, together with tunnel magnetoresistance, which we define as [7], $\mathrm{TMR}=\left(I_{S}^{A P}-I_{S}^{P}\right) / I_{S}^{P}$. For the magnetic molecule we assume that it is described by spin $S=2$ and magnetic anisotropy $D / U=0.05$. We do not impose any restrictions on the intrinsic exchange coupling $J$ and consider the case of both ferromagnetic (FM, $J>0)$ and antiferromagnetic (AFM, $J<0$ ) coupling. For comparison, we also present the results for $J=0$, which corresponds to a single quantum dot case [7]. This gives us the possibility to explicitly see how the presence of a large-spin molecule affects the transport characteristics.

Figure 2 shows the bias voltage dependence of the Andreev current flowing in both magnetic configurations of the device, together with the associated TMR, in the case of particle-hole symmetry point $\varepsilon=-U / 2$. In such situation the current is symmetric with respect to the bias reversal. Moreover, $I_{S}$ is relatively large since the

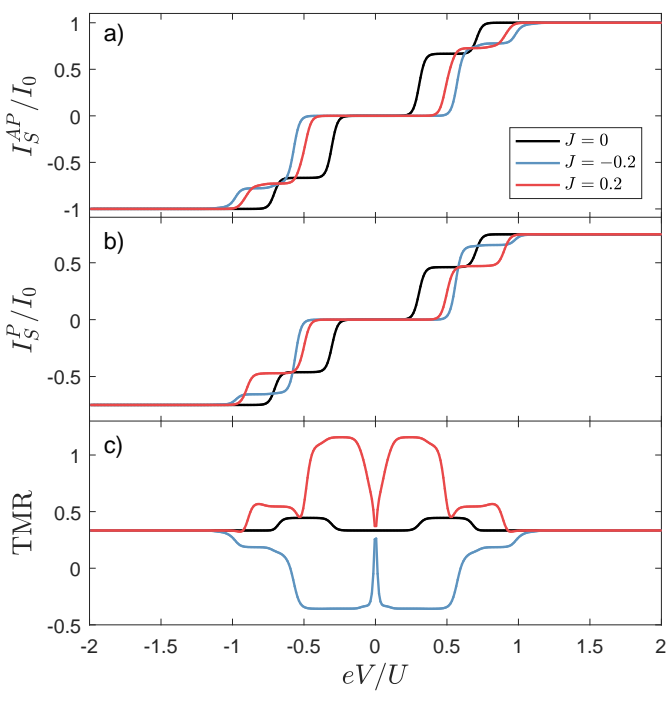

Fig. 2. The bias voltage dependence of the Andreev current in (a) the antiparallel and (b) parallel magnetic configuration, as well as (c) the TMR calculated for selected values of exchange interaction $J$. The parameters are: $\varepsilon=-0.5, \Gamma_{S}=0.4, \Gamma=0.01, T=0.025$, $D=0.05$, in units of $U \equiv 1$, and $S=2, p=0.5$. The current is plotted in units of $I_{0}=e \Gamma / \hbar$.

excitation energies to empty and doubly occupied molecular states are degenerate, such that the superconducting proximity effect is maximized. One can see that the coupling to the molecule changes the transport characteristics in comparison to the $J=0$ case. The conductance peaks in both configurations are displaced and the displacement magnitude is proportional to the coupling strength $J$. The reason for such a behavior is the modification of the Andreev bound states with $J$, which can be defined as excitation energies of a molecule decoupled from ferromagnetic leads [8].

At low bias voltage, the system is in the Coulomb blockade regime and only thermally-activated processes are allowed. The current starts flowing and increases in a step-wise fashion when the applied bias voltage exceeds certain threshold, such that Andreev bound states enter the window provided by the transport voltage. The current in the parallel configuration is smaller than that in the antiparallel configuration. This is associated with a mismatch in the spin-resolved densities of states. Since each Cooper pair involves two electrons of opposite spin, the minority spin subband is a bottleneck for transport in the parallel configuration and one generally finds $I_{S}^{P}<I_{S}^{A P}$. This is especially the case for larger bias voltages when all the Andreev states are active in transport and the current does not depend on $J$, such that $\mathrm{TMR}=p^{2} /\left(1-p^{2}\right)[7]$. However, when singleelectron states are mainly responsible for the Andreev current, the situation is more complex and one finds a strong dependence of the TMR on the type of exchange interaction, see Fig. 2(c). For ferromagnetic exchange interaction the TMR takes large positive values, while 
for antiferromagnetic $J$ interaction the TMR becomes negative. Large values of TMR indicate that transport is mainly due to crossed Andreev reflection (CAR) processes, in which Cooper pair electrons become split and leave the superconductor through two different junctions with ferromagnetic leads. On the other hand, low or negative values of the TMR indicate that direct Andreev reflection (DAR) processes are favored.

To understand the difference between the two types of exchange interaction, it is important to recall that in the case of parallel magnetic configuration a nonequilibrium spin accumulation can develop in the molecule. For $J>0$ and $V>0$, in the parallel configuration, the system is mainly occupied by the highest-weight spin multiplet $\left|S_{\text {tot }}, S_{\text {tot }}^{z}\right\rangle=\left|S+\frac{1}{2}, S+\frac{1}{2}\right\rangle$, where $S_{\text {tot }}$ is the total spin of SMM and $S_{\text {tot }}^{z}$ denotes its $z$ th component. On the other hand, in the antiparallel configuration the highest and lowest-weight spin multiplets are equally occupied, that is, there is no spin accumulation. Because of strong spin accumulation the Andreev current is suppressed in the parallel configuration compared to the antiparallel configuration where CAR processes involving majority spins of both leads are dominant. For antiferromagnetic exchange interaction and for positive bias, the occupation of the state $\left|S-\frac{1}{2}, S-\frac{1}{2}\right\rangle$ is close to unity. This situation is just opposite to the previous case. Now, one finds a suppression of the current in the antiparallel configuration compared to the parallel one, since the spin on the LUMO level is now mainly the minority spin of both leads. This results in the suppression of the TMR and even its sign change, see Fig. 2(c).

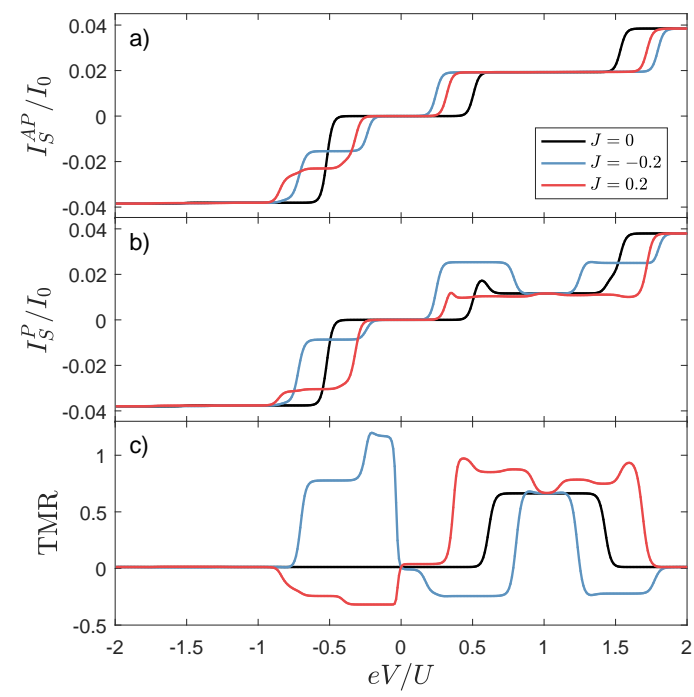

Fig. 3. The same as in Fig. 2 calculated for $\varepsilon=U / 2$.

Let us now analyze the system transport properties in the case of finite detuning from particle-hole symmetry point, $\varepsilon=U / 2$, see Fig. 3 . In the negative bias voltage region transport occurs mainly through states in which the LUMO level is not occupied. Then, ferromagnetic exchange interaction favors certain direction of the molecule spin. This direction is such that the current in the parallel configuration is favored, which results in negative TMR. For positive bias voltage, on the other hand, the ferromagnetic coupling makes the majority band spins occupying the LUMO level energetically more favorable, solidifying the spin accumulation and suppressing the current more effectively. Consequently, one observes an effect opposite to the case of $V<0$. Now, the current in the parallel configuration becomes suppressed, giving rise to large TMR, see Fig. 3(c).

The antiferromagnetic-coupling transport characteristics are qualitatively different from the FM exchange coupling case. The major difference is associated with the positive (negative) TMR in the negative (positive) bias region. Note that now the multiplet $\left|S-\frac{1}{2}, S-\frac{1}{2}\right\rangle$, which is a linear combination of both spin-up and spindown LUMO level spins, is relevant for transport. Because of that, the spin accumulation is not as strong as in the case of ferromagnetic $J$-coupling and one observes a completely opposite behavior of the TMR, see Fig. 3(c).

In summary, in this paper we have studied the spinresolved transport properties of a hybrid molecular junction with ferromagnetic and superconducting electrodes focusing on the subgap transport regime. We have shown that, depending on the type of intrinsic exchange interaction of the molecule, the TMR, which can be qualitatively associated with the amount of CAR processes, can take either negative or large positive values, depending on the transport voltage.

\section{Acknowledgments}

Work supported by National Science Centre in Poland through Project No. DEC-2013/10/E/ST3/00213.

\section{References}

[1] L. Bogani, W. Wernsdorfer, Nature Mat. 7, 179 (2008).

[2] C. Timm, F. Elste, Phys. Rev. B 73, 235304 (2006).

[3] M. Misiorny, I. Weymann, J. Barnaś, Phys. Rev. B 79, 224420 (2009).

[4] T. Domański, I. Weymann, M. Barańska, G. Górski, Sci. Rep. 6, 23336 (2016).

[5] H. Schoeller, G. Schön, Phys. Rev. B 50, 18436 (1994).

[6] M. Governale, M. Pala, J. König, Phys. Rev. B 77, 134513 (2008).

[7] I. Weymann, P. Trocha, Phys. Rev. B 89, 115305 (2014).

[8] B. Sothmann, D. Futterer, M. Governale, J. König, Phys. Rev. B 82, 094514 (2010). 